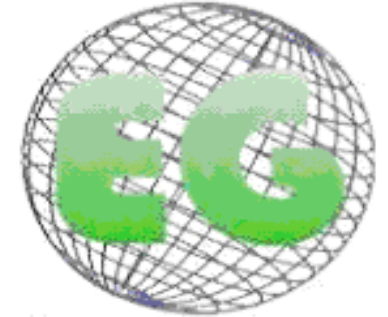

ISSN 1695-6141

$N^{\circ} 21$
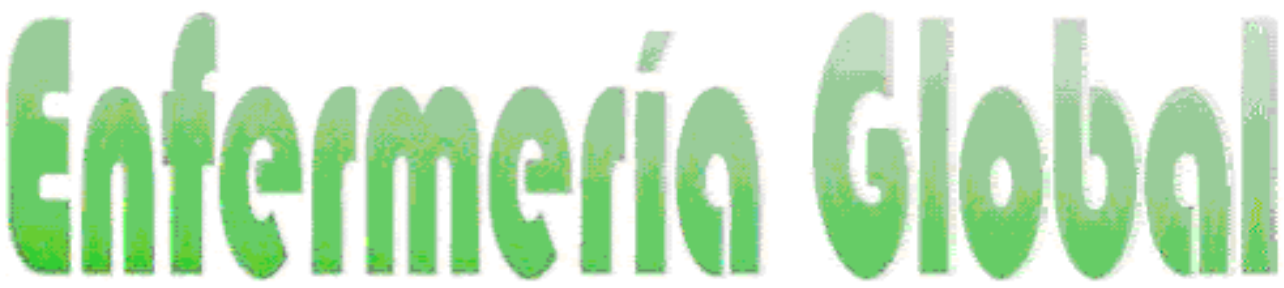

Revista electrónica trimestral de Enfermería

Enero 2011

www.um.es/egloball

CLÍNICA

\title{
HIGIENE DE MANOS COMO ESTRATEGIA FUNDAMENTAL EN EL CONTROL DE INFECCIÓN HOSPITALARIA: UN ESTUDIO CUANTITATIVO
}

HIGIENIZAC ÃO DAS MÃOS COMO ESTRATÉGIA FUINDAMENTAL NO CONTROLE DE INFECÇÃO HOSPITALAR: UM ESTUDO QUANTITATIVO

*Coelho, MS., *Silva Arruda, C., "*Faria Simões, SM.

*Enfermera. **Profesora Doctora Titular del Departamento de Fundamentos de Enfermería y Administración de la Escuela de Enfermería Aurora de Afonso Costa de la Universidad Federal Fluminense. Brasil.

(Trabajo presentado em el $60^{\circ}$ Congreso Brasileiro de Enfermería, Belo Horizontel MG y em el XI Congreso Brasileiro de Control de Infección y Epidemiologia Hospitalaria, Rio de Janeiro/ RJ como presentación a poster)

\author{
Palabras clave: Infección Hospitalaria, Lavado de Manos, Enfermería \\ Palavras chave: Infecção Hospitalar; Lavagem das mãos; Enfermagem. \\ Keywords: Cross infection, Handwashing, Nursing
}

\section{RESUMEN}

La infección hospitalaria sigue constituyendo un grave problema de salud pública en todo el mundo. Entre sus principales medidas de prevención y control está el lavado de manos, que según Semmelweis y Florence Nightingale, es un instrumento importante para la reducción de sus índices. Por lo tanto, este estudio descriptivo de carácter cuantitativo tuvo un enfoque destinado a analizar la frecuencia de lavado de manos por las enfermeras en un Hospital de la Universidad ubicado en la ciudad de Niteroi en el estado de Río de Janeiro, Brasil. Después de la aprobación en el Comité de Ética, se inició el estudio utilizando como una herramienta para la recogida de datos un cuestionario con preguntas abiertas y cerradas acerca de la práctica de lavarse las manos. En el análisis de los datos se determinó que $78 \%$ de los profesionales son mujeres. Con respecto a la edad, la mayor frecuencia se produjo en el grupo de 26 a 45 años con $60 \%$. Entre las categorías profesionales, $36 \%$ son enfermeras, $58 \%$ técnicos de enfermería y $6 \%$ auxiliares de enfermería. Con respecto a la formación o actualización en el ámbito de la infección hospitalaria, se encontró que $48 \%$ de los profesionales no lo han hecho. Sobre el lavado de manos, $98 \%$ respondió que lo hacen desde el principio hasta el final de la jornada, $96 \%$ entre uno u otro procedimiento y $86 \%$ después de la retirada de guantes. Entre los productos utilizados, se observó gran adhesión a agua y jabón, práctica citada por $92 \%$ de los profesionales, seguida por el alcohol en gel (44\%). Sin embargo, 
cuando se cuestionó acerca de cómo realizar la higiene, sólo $26 \%$ de los profesionales la describen y de manera sucinta. Después de la elaboración de esta investigación, se pudo concluir que, aunque los profesionales saben la importancia de lavarse las manos y dicen realizar con frecuencia la atención de los pacientes, para llevar a cabo efectivamente la técnica, se muestran con una baja adhesión, indicando la necesidad de una mayor participación en la formación y actualización sobre el tema.

\section{RESUMO}

As infecções hospitalares $(\mathrm{IH})$ ainda hoje constituem um grave problema de saúde pública mundial. Dentre suas principais medidas de prevenção e controle encontra-se a higienização das mãos o que segundo Semmelweis e Florence Nightingale é uma importante ferramenta para a redução de seus índices. Assim, esta pesquisa de caráter descritivo e abordagem quantitativa teve como objetivo analisar a freqüência de higienização das mãos por profissionais de enfermagem de um Hospital Universitário localizado no município de Niterói, no Estado do Rio de Janeiro, Brasil. Após aprovação no Comitê de Ética, iniciou-se o estudo utilizando como instrumento de coletas de dados um questionário com perguntas abertas e fechadas sobre a prática de higienização das mãos. Ao analisar os dados identificamos que $78 \%$ dos profissionais são do sexo feminino. No que se refere à faixa etária, a maior freqüência ocorreu no grupo de 26 a 45 anos com $60 \%$. Dentre as categorias profissionais, 36\% são enfermeiros, 58\% técnicos de enfermagem e 6\% auxiliares de enfermagem. No que tange a capacitação ou atualização na área de $\mathrm{IH}$, percebemos que $48 \%$ de profissionais não a tiveram. Em relação à higienização das mãos, $98 \%$ responderam que sempre as lavam ao longo do dia; $96 \%$ entre um procedimento e outro e $86 \%$ após a retirada das luvas. Entre os produtos utilizados, observamos grande aderência a água e sabão, sendo esta prática citada por $92 \%$ dos profissionais, seguida do álcool gel com $44 \%$. Porém, quando indagados sobre como realizavam a higienização, apenas $26 \%$ dos profissionais a descreveram e de modo sucinto. Após desenvolvimento deste estudo, podemos concluir que, apesar dos profissionais conhecerem a importância da higienização das mãos e afirmarem que a realizam freqüentemente durante a assistência ao paciente, ao desempenharem efetivamente a técnica demonstram uma baixa adesão, indicando ser necessário maior envolvimento profissional e atualização constante sobre o tema.

\section{ABSTRACT}

Hospital-acquired infections (HI) continue to constitute a serious public health issue worldwide. Among the main measures of prevention and control is hand hygiene, which, according to Semmelweis and Florence Nightingale, is an important tool to reduce $\mathrm{HI}$ rates. This research adopts a descriptive and quantitative approach to analyze the frequency of hand washing by healthcare staff of a university hospital located in Niterói, State of Rio de Janeiro, Brazil. After being approved by the Ethics Committee, the study began by collecting data through a questionnaire comprising open and closed questions about the practice of hand washing as an instrument of data collection. After analyzing the data collected, $78 \%$ of professionals have been identified as women. With regard to age, the highest frequency occurred in the group of individuals between 26 and 45 years at $60 \%$. Among the professional categories, $36 \%$ are nurses, $58 \%$ are practical nurses and $6 \%$, nursing assistants. Regarding training or upgrading in the area of $\mathrm{HI}$, we have observed that $48 \%$ of professionals have not acquired this. In relation to hand hygiene, $98 \%$ said they always wash them throughout the day, $96 \%$ said they do so in between procedures, $86 \%$, after removal of gloves. Among the products used for hand washing, we observed massive use of soap and water, a practice cited by $92 \%$ of the respondents, followed by alcohol gel at $44 \%$. But when asked about how they carried it out, only $26 \%$ of the professionals could describe it in detail. After this study, we conclude that, although health professionals know the importance of hand washing and argue that they frequently do it during care, just very few of them effectively perform the technique in a satisfactory way, which shows the need for greater professional involvement and constant update on the subject. 


\section{INTRODUCCIÓN}

Siendo el hospital lugar de cura y de atención de aquellos que poseen alguna necesidad de salud, debería proponer una asistencia humanizada y segura ante sus acciones asistenciales. Sin embargo, infelizmente, esta no es la realidad enfrentada actualmente. Poseemos aún altos índices de infecciones hospitalarias, comprometiendo la asistencia prestada en esos locales y haciéndonos reflexionar sobre la no concretización de prácticas seguras y eficaces de control coincidentes al saber epidemiológico.

Todavía hoy, la infección hospitalaria o nosocomial, constituye uno de los más graves problemas de salud pública, dado que sus altos índices de ocurrencia condicionan una elevación de la tasa de morbi-mortalidad y gasto oneroso del coste hospitalario, dificultando así la calidad del cuidar y la evolución del sistema de salud como todo (1).

Históricamente comprobada, el lavado de las manos se caracteriza como importante en la prevención a tales infecciones, siendo considerada la medida primordial contra la propagación de los microorganismos en el ámbito hospitalario (2).

La adopción de esta práctica posee importancia en el hecho de que gran porcentual de infecciones nosocomiales pueden ser evitadas, una vez que la mayoría de los microorganismos asociados a la microbiótica transitoria de las manos, o sea, aquella adquirida por el contacto con personas o materiales colonizados o infectados, podrían ser fácilmente eliminados a través de un adecuado lavado, dejando de ser condición básica para su diseminación (3).

A partir de estudios experimentales, en 1847, el médico húngaro Ignaz Philip Semmelweis (1818-1865), al depararse con los elevados índices de fiebre puerperal, postuló la interacción entre lavado de las manos e infección hospitalaria, de modo a instituir la obligatoriedad de la higiene de las manos con solución clorada entre la atención de cada paciente. En este momento, se evidenció la reducción drástica de la tasa de mortalidad materna (4).

Dentro de este contexto, la intervención de higiene de las manos propuesta por Semmelweis representó la primera evidencia de que el lavado de las manos ante el contacto con los clientes podría reducir los índices de infecciones asociadas a la asistencia a la salud (4).

Paralelo a esto, en el contexto de la Enfermería, Florence Nightingale se caracteriza como impulsora de este proceso. Dentro de esta perspectiva, preconizaba que cuando se instalaban, las enfermedades causaban la rotura de la dermis y consecuentemente se hacía puerta de entrada para microorganismos. De esta forma, siendo función de la enfermera la restauración de la salud, era inherente a esta, acciones de higiene de las manos a fin de promover la seguridad del cliente y propagación de un ambiente terapéutico seguro (5).

Reconociendo, por lo tanto, los escritos de Nightingale y Smmelweis como importantes vertientes de actuación en el campo de la prevención/contagio, se evidencia la necesidad de profesionales empeñados en el proceso salud-enfermedad y en la promoción de estrategias de control de las infecciones hospitalarias (6).

Con objetivo de validar la técnica y las orientaciones necesarias al lavado de las manos, el Centro de Control y Prevención de Enfermedades (CDC, Centers for Disease Control and Prevention), a partir de 1975, normativizó guías y directrices acerca de la práctica de higiene de las manos, recomendando las situaciones en las cuales se hace necesaria su utilización. 
Desde entonces, fueron publicadas nuevas guías de lavado y antisepsia con el objetivo de promover actualización/ capacitación sobre el tema. El más reciente, del año 2002, posibilitó gran repercusión en esta área, sustituyendo el término lavado de manos por higienización de las manos, caracterizando el abarcamiento de esta práctica (2).

Considerando la higienización de las manos como la remoción de los "microorganismos que colonizan las capas superficiales de la piel, así como el sudor, la oleosidad y las células muertas, retirando la suciedad propicia a la permanencia y a la proliferación de microorganismos" (2), y que uno de los mayores índices de transmisión de infección se da por las manos (7), su adopción debería ser contemplada por todos los profesionales de salud, para eso sería necesario la preparación adecuada de los mismos e inversiones en su concienciación (8).

Pero, observaciones del ambiente hospitalario reafirman la no uniformidad de conductas y rutinas referentes a su realización, y que a pesar de todas las evidencias y comprobaciones de la importancia a la adhesión a esta medida, las manos de los profesionales de salud aún se constituyen como el mayor vínculo de diseminación de las infecciones hospitalarias (9).

Entre los principales motivos relacionados al incumplimiento de esta práctica, según estudios recientes, está la falta de motivación, ausencia o inadecuación de lavabos o dispositivos de alcohol gel próximos a las camas, falta de materiales como jabón y alcohol además de toallas de papel y basureros, reacciones cutáneas ocasionadas por el uso de los productos recomendados, el gran número de tareas que sean realizadas ocasionando la falta de tiempo, irresponsabilidad y la ignorancia sobre la real importancia de las manos como medio de transmisión de microorganismos (8-10).

Siendo así, este estudio tiene como objetivo analizar la frecuencia de higienización de las manos por profesionales de enfermería del Hospital Universitario Antonio Pedro (HUAP), localizado en el municipio de Niterói, en el estado de Río de Janeiro, Brasil.

\section{MATERIAL Y MÉTODO}

Se trata de un estudio descriptivo con abordaje cuantitativo realizado en el Hospital Universitario Antônio Pedro, localizado en el municipio de Niterói, en el periodo de junio a septiembre de 2008.

Escogimos la investigación descriptiva, pues esta tiene como objetivo el levantamiento provisional del fenómeno que se desea estudiar de forma más detallada y estructurada, además de la obtención de informaciones acerca de un determinado producto posibilitando mayor familiaridad con el tema (11).

Participaron del estudio 50 profesionales de enfermería, escogidos de forma aleatoria, que suscribieron el término de consentimiento libre esclarecido y aceptaron participar del estudio. Todos son integrantes del cuadro de operarios del hospital universitario, actuando en sus diversas áreas de atención, como unidades de internación: Clínicas médicas y quirúrgicas, de Enfermedades Infecto-parasitarias (DIP) y Centro de Terapia Intensiva (CTI); en una de las unidades ambulatorias, el Centro de Diálisis; y en las unidades de emergencias: Pediátrica, Adulta y Trauma.

Como este es constituyente de un estudio mayor intitulado "Medidas de Precauciones Patrón, una Práctica Segura en Salud: un estudio exploratorio", de financiación de la 
Fundación de Amparo a la Investigación del Estado de Río de Janeiro (Faperj) y Consejo Nacional de Desarrollo Científico y Tecnológico (CNPq), fue sometido y aprobado por el Comité de Ética en Investigación de la Facultad de Medicina y Hospital Universitario Antônio Pedro/UFF en marzo de 2008.

Como instrumento de colecta de datos se utilizó un cuestionario con preguntas abiertas y cerradas, compuesto por una sesión de identificación de los sujetos y seguida por preguntas dirigidas a la temática abordada, o sea, que expresaran la práctica de la higienización de las manos realizadas por los profesionales a lo largo de su día de trabajo.

Anteriormente a su aplicación, el instrumento fue evaluado por una experta en el área a fin de que se alcanzara su mayor credibilidad, y los ajustes sugeridos fueron incorporados al cuestionario para mayor comprensibilidad de los entrevistados. Además de eso, se procedió a la ambientación priorizando la adecuación de las cuestiones formuladas a cuatro (4) profesionales de salud

Los cuestionarios fueron respondidos en el periodo comprendido entre los meses de agosto y septiembre del año de 2008, siendo estos distribuidos a los sujetos, que poseían el plazo máximo de devolución de siete días.

Los datos obtenidos fueron agrupados por su semejanza y posteriormente analizados e interpretados a la luz del método estadístico, siendo utilizado el programa Excel para la formulación de tablas y gráficos

\section{ANÁLISIS Y DISCUSIÓN DE LOS RESULTADOS}

Ante todos los cuestionarios recogidos y de la agrupación y análisis de los datos según la literatura pertinente, se originó la descripción y las discusiones que se siguen, divididas en dos grandes categorías: la identificación de los sujetos y la evaluación de la práctica de higienización en sí.

\section{Identificación de los Sujetos}

La muestra de este estudio fue compuesta por 18 ( $36 \%$ ) enfermeros, $29(58 \%)$ técnicos de enfermería y $3(6 \%)$ auxiliares de enfermería. Considerando que la proporción de técnicos de enfermería para enfermeros generalmente es mayor en los establecimientos de salud terciarios en Brasil y que hay una normativa del gobierno de este país para la extinción de la categoría auxiliar de enfermería, los números presentados son justificables

En lo que se refiere a la formación profesional, 11 (61\%) enfermeros, $12(41 \%)$ técnicos de enfermería y todos los auxiliares participantes del estudio la recibieron de un establecimiento público.

En la categoría tiempo de profesión, $36 \%$ de los sujetos poseían de 6 a 15 años de profesión, $34 \%$ con más de 15 años de experiencia, $28 \%$ con hasta 5 años, siendo que $2 \%$ no respondieron a esta cuestión. Estos datos caracterizan que gran parte de los sujetos puede ser considerada experimentada si evaluamos su tiempo de actuación profesional.

En relación al sexo, 39 (78\%) sujetos eran mujeres, lo que es natural ya que la profesión de enfermería es culturalmente femenina, a pesar de la creciente inserción del sexo masculino, al largo de esos años. La franja etaria entre 26 la 45 años fue la que más prevaleció, 
comprendiendo $60 \%$ de la población, seguido con $24 \%$ de mayores de 45 años y $14 \%$ de los que tenían de 18 a 25 años.

En cuanto a la temática principal del estudio, fue indagado a los profesionales participantes si habían recibido o realizado alguna capacitación sobre infección hospitalaria, 48\% respondieron que no, siendo que $34 \%$ no respondieron a este cuestionamiento.

Tabla 1: Identificación de los sujetos participantes del estudio. Medidas de precaución padrón y la práctica segura en salud: en estudio exploratorio. Niterói, 2008

\begin{tabular}{|l|l|l|}
\hline \hline & $\mathrm{N}^{0}$ & $\%$ \\
\hline Sexo & & \\
\hline Masculino & 39 & 78 \\
\hline & 11 & 39 \\
\hline Edad & & \\
\hline $18-25$ años & & \\
\hline $26-45$ años & 07 & 14 \\
\hline$>45$ años & 30 & 60 \\
\hline No respondió & 12 & 24 \\
\hline & 01 & 2 \\
\hline Formación profesional: & & \\
\hline Enfermero & & \\
\hline Técnico en enfermería & 18 & 36 \\
\hline Auxiliar en enfermería & 29 & 58 \\
\hline & 03 & 6 \\
\hline Tiempo de profesión & & \\
\hline Hasta 5 afios & & \\
\hline 6 a 15 años & 14 & 28 \\
\hline$>15$ años & 18 & 36 \\
\hline & 17 & 34 \\
\hline & 01 & 2 \\
\hline Capacitación en IH & & \\
\hline Si & & \\
\hline No & 9 & 18 \\
\hline No Respondió & 24 & 48 \\
\hline \hline & 17 & 34 \\
\hline & & \\
\hline
\end{tabular}

\section{Evaluando la Higienización de las manos}

Como vimos, la higienización de las manos se constituye como medida primaria para la prevención de las infecciones hospitalarias, una vez que las manos se caracterizan como principal herramienta de transmisión de los microorganismos (12). Pero, la falta de adhesión de los profesionales de salud a esta práctica acarrea la necesidad de reformulación cultural, a fin de valorarse la seguridad y la calidad de asistencia.

Siendo esta evidencia recomendada a todo profesional de salud, elaboramos 3 cuestiones sobre la temática, con el objetivo de abordar esta técnica y comprender la dimensión que los profesionales poseen sobre el tema. En la primera cuestión, reflexionamos sobre la práctica de higienización de las manos a lo largo del día. 
Como resultados, evidenciamos que $98 \%$ de los profesionales siempre la realizan y $2 \%$ la realizan a veces. De esa forma, según la literatura, constatamos que los entrevistados se muestran implicados con la realización de esta medida y con la reducción de la propagación de las infecciones relacionadas a la asistencia.

\section{Gráfico 1 : frecuencia de higienización de las manos a lo largo del día} Niterói, 2008

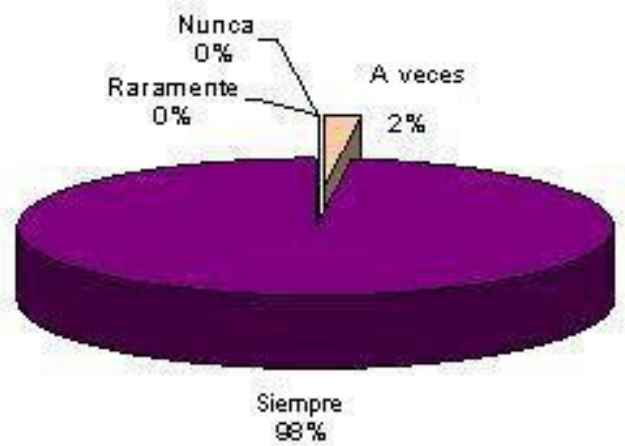

Sin embargo, nuestra práctica académica evidencia una distorsión ante este resultado, una vez que no presenciamos tal implicación. Además de eso, la estructura física presentada en los sectores condiciona la baja adhesión de los profesionales, visto que en las unidades, muchas veces hay solamente un lavabo para la realización de esta higiene, y este se encuentra sólo en el puesto de enfermería siendo inexistente en las enfermerías, o sea, próximo a las camas.

Con relación a la higienización de las manos entre un procedimiento y otro, percibimos que $96 \%$ de los entrevistados siempre la hacen, mientras solamente $4 \%$ la hacen a veces. Así, aún en este sentido, preguntamos cómo y con qué productos estos realizan esta técnica.

Gráfico 2: frecuencia de higienización de las manos durante procedimientos de asistencia al cliente. Niterói, 2008

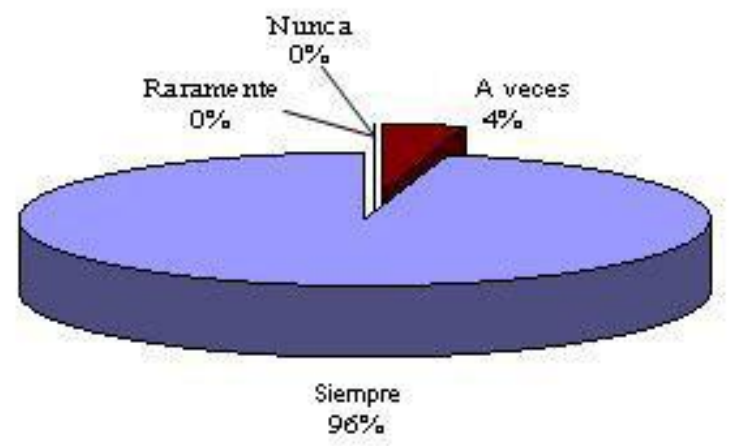

En cuanto a los productos, observamos gran adherencia al agua y jabón, siendo esta práctica citada $92 \%$, seguida del alcohol gel con $44 \%$. La otra alternativa de respuesta fue degermante con $4 \%$, además de eso 2 profesionales (4\%) no respondieron. 
Gráfico 3 : Materiales más utilizados en la higienización de las manos por los sujetos del estudio. Niterói, 2008

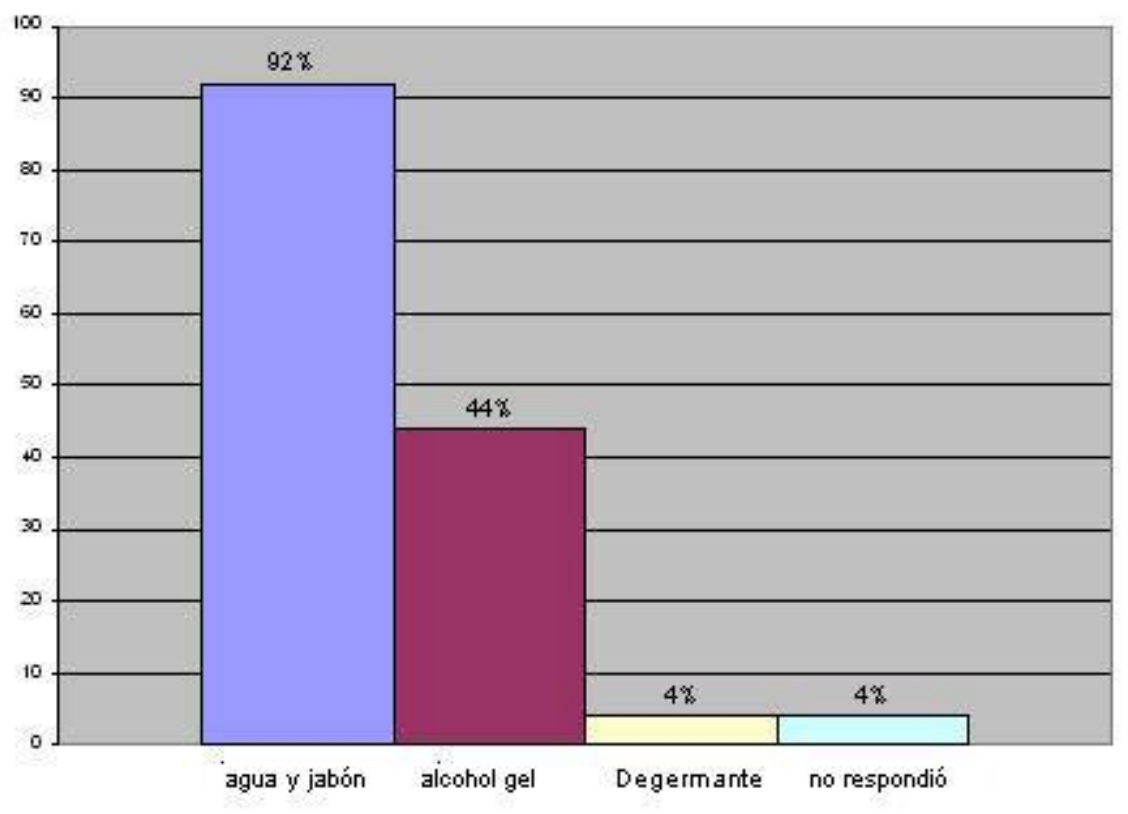

En este sentido, percibimos una prevalencia de la variable agua y jabón, a pesar de esta ser recomendada entre un procedimiento y otro solamente cuando las manos estuvieran aparentemente sucias.

La higiene de las manos es preconizada cuando hay suciedad aparente, contaminación por fluidos y sangre, después de uso de los sanitarios, antes y después de la alimentación, al iniciar el turno de trabajo, antes de la preparación y manipulación de medicamentos, después de varias aplicaciones de las preparaciones alcohólicas y antes y después de contacto con paciente colonizado o infectado por C. difficile (13).

En relación la forma como realizaban la higienización, sólo $26 \%$ de los profesionales la describieron de modo general y sucinto, no especificando las fases inherentes a la técnica para que esta tenga eficacia. Así, podemos apuntar que no hubo una interpretación adecuada de este cuestionamiento en el instrumento utilizado, y que el mismo debería ser presentado en la forma de diagrama para una mejor visualización y comprensión.

Además de eso, para que la práctica de higienización de las manos sea efectiva, es esencial la planificación de intervenciones en los servicios de salud que viabilicen discusiones sobre el tema, ya que los profesionales afirmaron su realización, pero se mostraron inseguros o incapaces de describir la técnica utilizada.

Cuando indagamos sobre el lavado de las manos después de la retirada de los guantes, ya que la utilización de los mismos no excluye la obligatoriedad de la higienización de las manos, $86 \%$ de los entrevistados respondieron siempre higienizarla, mientras que $14 \%$ afirmaron realizarla a veces. 
Gráfico 4: frecuencia de higienización de las manos después de la utilización de los guantes. Niterói, 2008

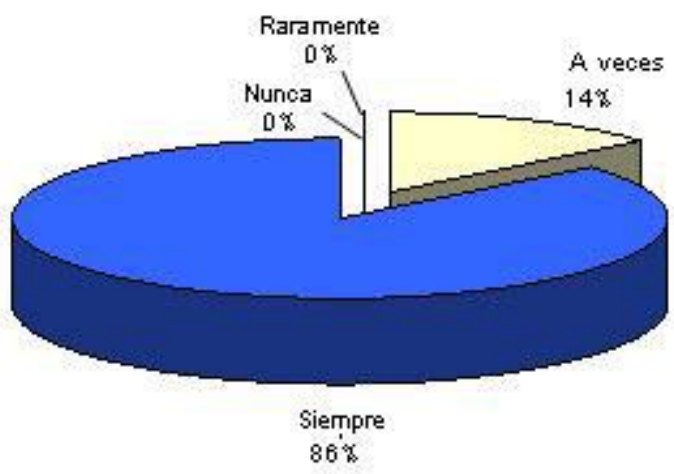

Simultáneamente a esta cuestión, también preguntamos con qué estos la higienizan. En esta cuestión, constatamos que el agua y jabón fueron mencionados en $92 \%$ de las respuestas, el alcohol gel en $32 \%$ y el degermante en $4 \%$, así como $2 \%$ no respondieron.

Gráfico 5: Materiales utilizados en el lavado de manos después del uso de los guantes. Niterói, 2008

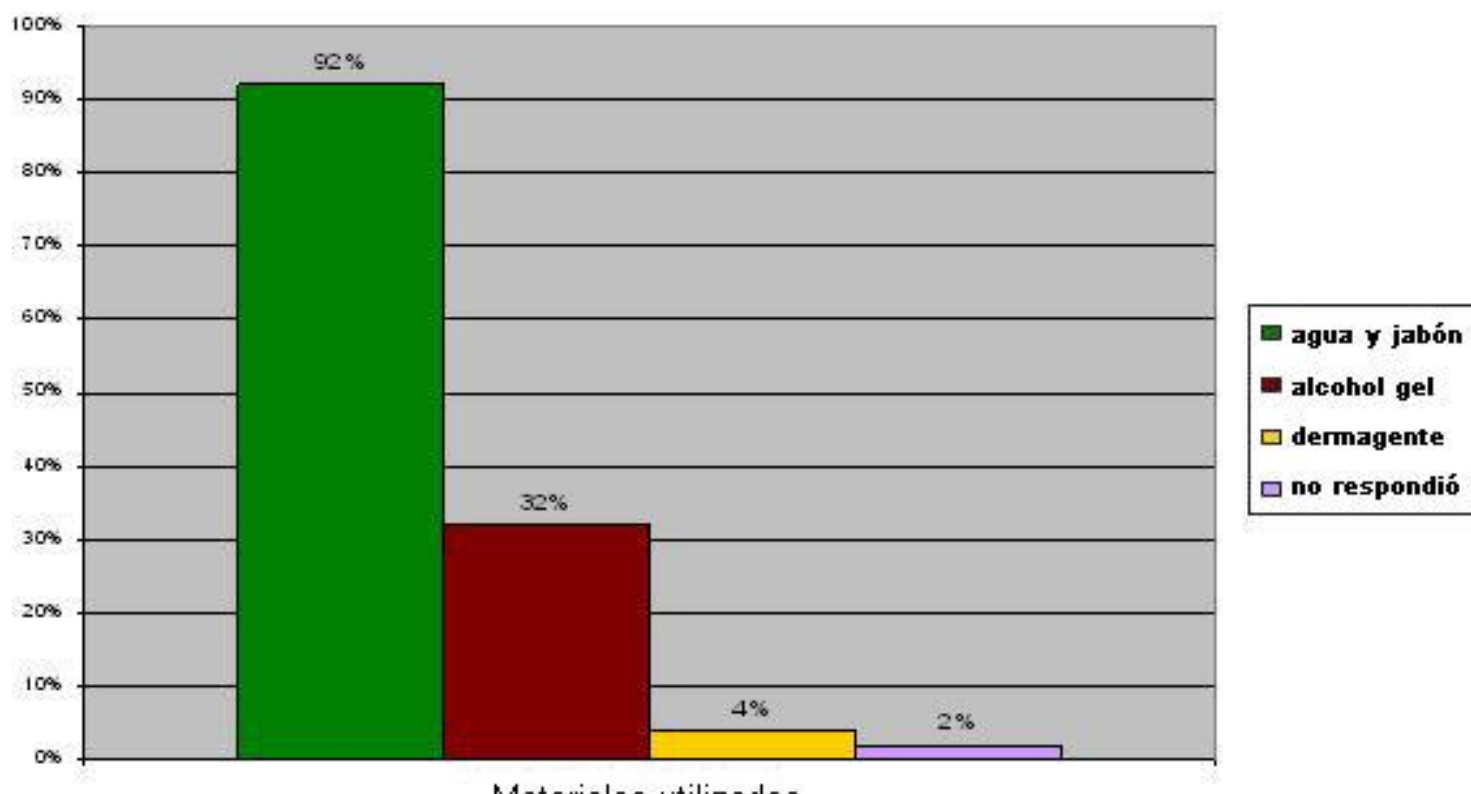

Materiales utilizados

Similar a la higienización entre un procedimiento y otro, se recomienda que después de la retirada de guantes se utilice el alcohol gel, sin embargo, entendemos que es coherente que el talco existente en el guante proporcione incomodidad y los profesionales de salud prefieran la higiene de las manos con agua corriente y jabón.

Al final de los cuestionamientos, fue posible notar que los sujetos poseen gran percepción sobre el acto que abarca la higienización de las manos en su práctica profesional y por eso respondieron positivamente a todas las alternativas levantadas. 
En estudio realizado en el mismo hospital, se reveló que esta preocupación es similar en relación a los equipamientos de protección individual, donde datos demostraron la disponibilidad de estos en las unidades y la concienciación de los profesionales por la importancia de su uso (14).

Mientras tanto, al analizar la realidad en la que estamos insertados y la asistencia prestada, tenemos conciencia de que esta no coincide con los resultados referidos en estos estudios, lo que nos causa gran inquietud

Después del análisis preliminar, las investigadoras entendieron ser oportuno ampliar el método del estudio, insertando la observación sistemática para la validación de los datos iniciales.

\section{CONSIDERACIONES FINALES}

Debemos entender la prevención y el control de las infecciones hospitalarias como responsabilidad individual y colectiva, pues sin la asimilación y la implementación correcta de los procedimientos ejecutados por quien presta el cuidado al paciente, esta continuará siendo una traba a la calidad en la prestación de los servicios de salud

Así, evidenciamos la necesidad de actualizaciones, como forma de promover mayor discusión sobre el asunto, por ejemplo, abordando la realización de la técnica correcta de higienización de las manos, con objetivo de aclarar dudas; aplicando posteriores evaluaciones para que la estrategia utilizada sea siempre perfeccionada o modificada, a fin de que se alcance el objetivo propuesto (15).

El enfermero, pieza fundamental en el cuidar, debe estar en constante interacción con el proceso de educación en salud, procurando con sus acciones a atender mejor a los pacientes y promover estrategias de transformaciones en el escenario de la asistencia (16).

Además de eso, debe estimular la concienciación del equipo ante la seguridad del ambiente, del paciente y del propio profesional en su cotidiano hospitalario. Esto porque sólo el conocimiento del fenómeno infección hospitalaria y de sus correspondientes medidas preventivas, no garantiza la adhesión y eficacia de una atención en salud

Resaltamos también que para alcanzar el éxito de esta concienciación, se debe primordialmente establecer la implicación de esos profesionales, promoviendo su debido perfeccionamiento y estimulando su motivación, en las diferentes interfaces de la atención a los pacientes, siendo estrategias llaves en la disminución de los índices de infección hospitalaria y en la adopción de prácticas correctas y seguras.

\section{REFERENCIAS BIBLIOGRÁFICAS}

1- Pereira MS, Souza ACD, Tipple AFV, Prado MA. A infecção hospitalar e suas implicações para o cuidar da enfermagem. Texto \& contexto enferm. 2005 Abr- Jun;14(2):250-7.

2- Souza FC, Rodrigues IP, Santana HT. Perspectiva histórica. In: Agência Nacional de Vigilância Sanitária, Ministério da Saúde. Segurança do paciente: higienização das mãos. Brasília (DF); 2008. p.11-15. 
3- Oliveira AC. Infecções hospitalares: repensando a importância da higienização das mãos no contexto da multirresistência. Rev Min Enf. 2003 Dez; 7(2):140-44.

4- Centers for Disease Control and Prevention. Guideline for hand hygiene in health-care settings: recommendations of the healthcare infection control practices advisory committee and the HICPAC/SHEA/APIC/IDSA hand hygiene task force. MMWR. 2002; 51:1-56.

5- Lobo ML. Florence Nightingale. In: George JB. Teorias de enfermagem: os fundamentos à prática profissional. Ed 4. Porto Alegre: Artes Médicas Sul; 2000. p.33-44.

6- Carraro TE. Os Postulados de Nightingale e Smmelweis: poder vital/vida e prevenção/contágio como estratégias para a evitabilidade das infecções. Rev. latinoam. Enferm. 2004 Jul-Ago;12(4):650-7.

7- Agência Nacional de Vigilância Sanitária. Ministério da Saúde. Gerência-Geral de Tecnologia em Serviços de Saúde. Unidade de Controle de Infecção em Serviços de Saúde. Tema Nacional: Lavagem das Mãos [homepage na Internet]. Brasília: Ministério da Saúde; atualizada em 01/12/2008. Acesso em 16/07/2008. Disponível em: $<$ http://www.ccih.med.br/tema-nacional-2001.htm>

8- Neves ZCP, Tipple, AFV, Souza ACS, Pereira MS, Melo DS, Ferreira LR. Higienização das mãos: o impacto de estratégias de incentivo à adesão entre profissionais de saúde de uma unidade de terapia intensiva neonatal. Rev. latinoam. Enferm. 2006 Jul-Ago;14(4):546552.

9- Scheidt KLS, Carvalho M. Avaliação Prática da Lavagem das Mãos Pelos Profissionais de Saúde em Atividades Lúdico-Educativas. Rev. enferm. UERJ. 2006 Abr-jun; 14(2):221-5.

10- Oliveira AC, Werly A, Ribeiro, MR, Neves FAC, Junior FFF, Junior FSO. Adesão à higienização das mãos entre a equipe multiprofissional de uma unidade de terapia intensiva infantil - um estudo transversal e descritivo. Online braz. j. nurs. (Online) [periódico na Internet].2007. Acesso em 18/12/08; 6(1): 12p. Disponível em: < http://www.uff.br/objnursing/index.php/nursing/rt/printerFriendly/732/166>.

11- Oliveira, SL. Tratado de metodologia científica. São Paulo: Pioneira; 1997:320p.

12- Martins MA. Manual de Infecção Hospitalar: Epidemiologia, Prevenção e Controle. $2^{\circ} e d$. Belo Horizonte: MEDSI Editora Médica e Científica Ltda; 2001:1116p.

13- Amorim AB, Santana HT, Sousa FC, Barcellos RMG. Equipamentos e Insumos Necessários para Higienização das Mãos. In: Agência Nacional de Vigilância Sanitária, Ministério da Saúde. Segurança do paciente: higienização das mãos. Brasília: 2008. 53-5.

14- Simões SMF, Coelho MS, Arruda CS. Equipamento de Proteção Individual e a Prática Segura em Saúde. In: Anais do The Brazilian Journal of Infections Diseases; 2008 nov. 12 (Suppl 3): 238. Rio de Janeiro; 2008.

15- Sánchez-Payá J., Fuster-Pérez M., García-González C., Gracia-Rodríguez R. M., García-Shimizu P., San Juan-Quíles A. et al . Evaluación de un programa de actualización de las recomendaciones sobre la higiene de manos. Anales Sis San Navarra. [periódico na Internet]. 2007 Dez . Acesso em 18/12/08; 30(3): 343-352. Disponivel 
em:<http://scielo.isciii.es/scielo.php?script=sci_arttext\&pid=\$113766272007000500003\&lng= es\&nrm=iso.>.

16- Dias LMC, Tocantins FR, Silva TJ. A Educação Permanente Como Instrumento de Transformação nas Práticas do Assistir/Cuidar da Enfermagem. Enferm. glob. (Online) [periódico na Internet].2007 May. Acesso em 18/12/08; 10:1-8. Disponível em: <http://revistas.um.es/eglobal/article/viewFile/216/252. 